\title{
GLOBAL DEFENSIVE $k$-ALLIANCES IN DIRECTED GRAPHS: COMBINATORIAL AND COMPUTATIONAL ISSUES
}

\author{
Doost Ali Mojdeh ${ }^{1}$, Babak Samadi ${ }^{1}$ And Ismael G. Yero ${ }^{2, *}$
}

\begin{abstract}
In this paper we define the global defensive $k$-alliance (number) in a digraph $D$, and give several bounds on this parameter with characterizations of all digraphs attaining the bounds. In particular, for the case $k=-1$, we give a lower (an upper) bound on this parameter for directed trees (rooted trees). Moreover, the characterization of all directed trees (rooted trees) for which the equality holds is given. Finally, we show that the problem of finding the global defensive $k$-alliance number of a digraph is NP-hard for any suitable non-negative value of $k$, and in contrast with it, we also show that finding a minimum global defensive $(-1)$-alliance for any rooted tree is polynomial-time solvable.
\end{abstract}

Mathematics Subject Classification. 05C20, 05 C69.

Received October 4, 2018. Accepted May 2, 2019.

\section{INTRODUCTION AND PRELIMINARIES}

Alliances in graphs were first described by Kristiansen et al. [8], where alliances were classified into defensive, offensive or powerful. After this seminal paper, the issue has been intensively studied. Remarkable examples are the articles $[14,15]$, where alliances were generalized to $k$-alliances. To see more information on alliances in graphs we suggest the recent surveys $[2,11,19]$ and references cited therein.

Alliances exist in several ways in real world. Roughly speaking, an alliance can be understood as a collection of elements sharing similar objectives or having similar properties among all elements of the collection. Examples of alliances could be a group of people united by a common friendship, a group of plants belonging to the same botanical family, a group of Twitter users following or being followed among themselves, or a group of Facebook users sharing a common activity. For instance, Facebook can be seen as an enormous network (or graph) in which each user is a vertex and two vertices are connected if they are "friends", in the sense of the system. With this idea, an alliance in Facebook can be realized as a collection of users (or vertices) having more "friends" inside the collection than outside. Analogously, Twitter can be understood as a graph in which each user is a vertex and two vertices are adjacent if at least one of them is "following" the other one. Hence, an alliance in Twitter can be understood as a collection of users following (or being followed) more users (by more users) inside the collection than outside. However, if we deeply observe such models, there is a lack of reality, since the connections are frequently "not in both directions". That is, if we consider the Twitter network, one can

Keywords. Global defensive $k$-alliance number, dominating set, $k$-order-sum number, rooted tree, directed tree.

1 Department of Mathematics, University of Mazandaran, Babolsar, Iran.

2 Departamento de Matemáticas, Universidad de Cádiz, Algeciras, Spain.

*Corresponding author: ismael.gonzalez@uca.es 
see that a user $A$ can follows another user $B$, but this does not mean that also $B$ will follow $A$. Thus, the "connection" is only in one "direction". According to this fact, we could think into describe alliances whether the connection between users is not flat, namely, there is a direction.

Applications of alliances can be found in the Ph.D. Thesis [13] where the author studied problems of partitioning a graph into alliances and its application to data clustering. On the other hand, defensive alliances represent the mathematical model of web communities, by adopting the definition of Web Community proposed by Flake et al. [3], "a Web Community is a set of web pages having more hyperlinks (in either direction) to members of the set than to non-members". And we again can notice here the lack of reality described above, since the hyperlinks are always having a direction (they go from one point to another).

Based on all these facts, we are now addressed to consider directions in the connections between vertices in a network in order to study the alliances of such network. In other words, we are aimed to study the topic of alliances in directed graphs (digraphs).

Throughout this paper, we consider $D=(V(D), A(D))$ as a finite digraph with vertex set $V=V(D)$ and arc set $A=A(D)$ with neither loops nor multiple arcs (although pairs of opposite arcs are allowed). Also, $G=(V(G), E(G))$ stands for a simple finite graph with vertex set $V(G)$ and edge set $E(G)$. We use $[1,18]$ as references for some very basic terminology and notation in digraphs and graphs, respectively, which are not explicitly defined here.

For any two vertices $u, v \in V(D)$, we write $(u, v)$ as the $\operatorname{arc}$ with direction from $u$ to $v$, and say $u$ is adjacent to $v$, or $v$ is adjacent from $u$. Given a subset $S$ of vertices of $D$ and a vertex $v \in V(D)$, the in-neighborhood of $v$ from $S$ (out-neighborhood of $v$ to $S$ ) is $N_{S}^{-}(v)=\{u \in S \mid(u, v) \in A(D)\}\left(N_{S}^{+}(v)=\{u \in S \mid(v, u) \in A(D)\}\right)$. The in-degree of $v$ from $S$ is $\operatorname{deg}_{S}^{-}(v)=\left|N_{S}^{-}(v)\right|$ and the out-degree of $v$ to $S$ is $\operatorname{deg}_{S}^{+}(v)=\left|N_{S}^{+}(v)\right|$. Moreover, $N_{S}^{-}[v]=N_{S}^{-}(v) \cup\{v\}\left(N_{S}^{+}[v]=N_{S}^{+}(v) \cup\{v\}\right)$ is the closed in-neighborhood (closed out-neighborhood) of $v$ from (to) $S$. In particular, if $S=V(D)$, then we simply say (closed) (in or out)-neighborhood and (in or out)degree, and write $N_{D}^{+}(v), N_{D}^{-}(v), N_{D}^{+}[v], N_{D}^{-}[v], \operatorname{deg}_{D}^{+}(v)$ and $\operatorname{deg}_{D}^{-}(v)$ instead of $N_{V(D)}^{+}(v), N_{V(D)}^{-}(v), N_{V(D)}^{+}[v]$, $N_{V(D)}^{-}[v], \operatorname{deg}_{V(D)}^{+}(v)$ and $\operatorname{deg}_{V(D)}^{-}(v)$, respectively (we moreover remove the subscripts $D, V(D)$ if there is no ambiguity with respect to the digraph $D$ ). Let $S \subseteq V(D)$ and $u \in S$. A vertex $v$ in $V(D)$ is called a private out-neighbor of $u$ with respect to $S$ if $N^{-}[v] \cap S=\{u\}$. The set of all private out-neighbors of $u$ with respect to $S$ is denoted by $p n^{+}(u, S)$. Given two sets $A$ and $B$ of vertices of $D$, by $(A, B)_{D}$ we mean the sets of arcs of $D$ going from $A$ to $B$. For a graph $G, \Delta(G)$ and $\delta(G)$ represent the maximum and minimum degrees of $G$. In addition, for a digraph $D, \Delta^{+}(D)$ and $\delta^{+}(D)$ represent maximum and minimum out-degrees and $\Delta^{-}(D)$ and $\delta^{-}(D)$ represent the maximum and minimum in-degrees of $D$. If there is no confusion, then we avoid writing the graph $G$ or the digraph $D$ (for instance, we would simply write $\Delta^{-}$for the maximum in-degree instead of $\left.\Delta^{-}(D)\right)$.

We denote the converse of a digraph $D$ by $D^{-1}$, obtained by reversing the direction of every arc of $D$. A digraph $D$ is connected if its underlying graph is connected. A rooted tree is a connected digraph with a vertex of in-degree 0 , called the root, such that every vertex different from the root has in-degree 1 . In a rooted tree, the vertex of out-degree 0 is called a leaf and its in-neighbor is a support vertex. A directed tree is an orientation of a tree. A directed star $S_{n}$ on $n$ vertices is a rooted tree of order $n$ in which its root is adjacent to all other vertices.

Given a graph $G$, a set $S \subseteq V(G)$ is a dominating set in $G$ if each vertex in $V(G) \backslash S$ is adjacent to at least one vertex in $S$. The domination number $\gamma(G)$ is the minimum cardinality of a dominating set in $G$. For more information about this concept the reader can consult [6]. The concept of domination in digraphs was introduced by $\mathrm{Fu}$ [4] as follows. A subset $S$ of vertices of a digraph $D$ is called a dominating set if every vertex in $V(D) \backslash S$ is adjacent from a vertex in $S$. The domination number $\gamma(D)$ of $D$ is the minimum cardinality of a dominating set in $D$.

As already mentioned, Kristiansen et al. [8] introduced the concept of defensive alliance in graphs as follows. A non-empty set of vertices $S \subseteq V(G)$ is called a defensive alliance if for every $v \in S, \operatorname{deg}_{S}(v) \geq \operatorname{deg}_{\bar{S}}(v)-1$, where $\bar{S}=V(G) \backslash S$. They called it global if $S$ is a dominating set. The concept of (global) defensive alliance 
in a graph $G$ was generalized in [13-16] to that of (global) defensive $k$-alliance as a dominating set $S$ of $G$ for which $\operatorname{deg}_{S}(v) \geq \operatorname{deg}_{\bar{S}}(v)+k$, for all $v \in S$, and where $k \in\{-\Delta(G), \ldots, \Delta(G)\}$. The global defensive $k$-alliance number of $G$, denoted by $\gamma_{k}^{d}(G)$, is the minimum cardinality of a global defensive $k$-alliance in $G$.

Since then a high number of papers on different versions of alliances in graphs have been published in the literature and some interesting real-world applications can be found among them (the reader can refer to $[8,12,14]$ for instance). To have a better overview on all the information about alliances we suggest the fairly complete surveys $[2,11,19]$.

Definition 1.1. Let $D$ be a digraph and let $k \in\left\{-\Delta^{+}(D), \ldots, \Delta^{+}(D)\right\}$ be an integer. A set of vertices $S \subseteq V(D)$ is called a global defensive $k$-alliance in $D$ provided that every vertex in $V(D) \backslash S$ is adjacent from a vertex in $S$ and $\operatorname{deg}_{S}^{+}(v) \geq \operatorname{deg}_{\bar{S}}^{+}(v)+k$, for all $v \in S$. The global defensive $k$-alliance number, denoted $\gamma_{k}^{d}(D)$, is defined as the minimum cardinality of a global defensive $k$-alliance in $D$. We call the global defensive $(-1)$-alliance (number) just global defensive alliance (number), for short.

Since we are dealing with digraphs in this work, we clearly have directions in the connections. Throughout all this exposition, alliances are considered for "out" directions. One would then think as natural to also deal with alliances with an opposed direction. In this sense, the main concept of this paper (the global defensive $k$-alliance number) could be defined as follows for an opposed version with respect to the arcs.

Let $D$ be a digraph and let $k \in\left\{-\Delta^{-}(D), \ldots, \Delta^{-}(D)\right\}$ be an integer. A set of vertices $S \subseteq V(D)$ is called a global out-defensive $k$-alliance in $D$ provided that every vertex in $V(D) \backslash S$ is adjacent to a vertex in $S$ and $\operatorname{deg}_{S}^{-}(v) \geq \operatorname{deg}_{\bar{S}}^{-}(v)+k$, for all $v \in S$. The global out-defensive $k$-alliance number, denoted $\gamma_{k}^{d-}(D)$, is defined as the minimum cardinality of a global out-defensive $k$-alliance in $D$. In concordance, the main concept of this work could be also called global in-defensive $k$-alliance. In consequence, the parameters global out-defensive $k$-alliance (number) and the global in-defensive $k$-alliance (number) of a digraph $D$ could be of interest for a possible research line. However, it is easy to check that $\gamma_{k}^{d-}(D)=\gamma_{k}^{d}\left(D^{-1}\right)$, for any digraph $D$. Based on this fact, we only center our attention on the out version. We recall that for a graph $G$, the complete biorientation $\overleftrightarrow{G}$ of $G$ is a digraph obtained from $G$ by replacing each edge $x y \in E(G)$ by the pair of arcs $(x, y)$ and $(y, x)$.

Our first observation shows an important relationship which exists between alliances in graphs and in digraphs. We consider a graph $G$ and the digraph $D$ obtained as the complete biorentation of $G$. It is easy to see that $G \longrightarrow D$ is an one-to-one correspondence between the collection $\mathbb{G}$ of all graphs and the collection $\mathbb{D}$ of digraphs for which there exist two $\operatorname{arcs}(x, y)$ and $(y, x)$ between vertices $x$ and $y$ in $V(G)=V(D)$. Now, we can also readily see that a set of vertices $S$ is a global defensive $k$-alliance in $G$ if and only if $S$ is a global defensive $k$-alliance in $D$, implying the following result.

Remark 1.2. For any graph $G$ and any integer $k \in\{-\Delta(G), \ldots, \Delta(G)\}, \gamma_{k}^{d}(G)=\gamma_{k}^{d}(D)$, where $D$ is the complete biorientation of $G$.

Clearly, such a relationship is very useful when we deal with digraphs for which an arc $(u, v)$ exists if and only if the $\operatorname{arc}(v, u)$ also exists. However, this is a small subfamily of the whole class of digraphs, and therefore, our study on general digraphs is of interest, and clearly independent of the study of defensive $k$-alliances in graphs.

In this paper, we give several lower bounds on $\gamma_{k}^{d}(D)$, as for example we prove that $\gamma_{k}^{d}(D) \geq 2 n /\left(\Delta^{+}-k+2\right)$, and give the characterization of all digraphs $D$ for which the equality holds. Also, we bound $\gamma_{k}^{d}(D)$ from below by $\min \left\{t \mid d_{1}^{+}+\ldots+d_{t}^{+} \geq 2 n+(k-2) t\right\}$. We prove that $(n+1) / 3$ is a lower bound on $\gamma_{-1}^{d}(T)$ for a directed tree $T$ of order $n$, and $\gamma_{-1}^{d}(T) \leq\lfloor(n+\gamma(T)) / 2\rfloor$ for all rooted tree $T$ of order $n$. Moreover, we characterize all directed trees and rooted trees $T$ attaining these bounds. We end the exposition with a computational study on computing the global defensive $k$-alliance number of digraphs.

From now on, given any parameter $\eta$ in a graph $G$ (or a digraph $D$ ), a set of vertices of cardinality $\eta(G)$ (or $\eta(D))$ is called a $\eta(G)$-set (or $\eta(D)$-set). Moreover, since the integer $k$ always fits in the interval $\left\{-\Delta^{+}(D), \ldots, \Delta^{+}(D)\right\}$, we will skip this assumption and, unless specifically stated another situation, we will always consider $k \in\left\{-\Delta^{+}(D), \ldots, \Delta^{+}(D)\right\}$. Also, throughout the whole article the inequality $\operatorname{deg}_{S}^{+}(v) \geq \operatorname{deg}_{\bar{S}}^{+}(v)+k$, satisfied by a vertex $v \in S$ will be called the global defensive $k$-alliance condition. 


\section{General Digraphs}

In order to begin our exposition we need to introduce one family of digraphs. We construct the family $\Omega$ of digraphs $D$ as follows. Let $D^{\prime}$ be a digraph with each vertex of out-degree $r \geq k$. We add $r-k$ private out-neighbors lying outside $V\left(D^{\prime}\right)$, for every $v \in V\left(D^{\prime}\right)$. Let

$$
V(D)=V\left(D^{\prime}\right) \cup\left(\bigcup_{v \in V\left(D^{\prime}\right)} p n^{+}\left(v, V\left(D^{\prime}\right)\right)\right) .
$$

Add some arcs among the vertices in $\overline{V\left(D^{\prime}\right)}$ and some $\operatorname{arcs}(u, v)$, for some $u \in \overline{V\left(D^{\prime}\right)}$ and $v \in V\left(D^{\prime}\right)$, such that $\operatorname{deg}^{+}(u) \leq 2 r-k$, for all $u \in \overline{V\left(D^{\prime}\right)}$. Clearly, every vertex in $\overline{V\left(D^{\prime}\right)}$ is adjacent from exactly one vertex in $V\left(D^{\prime}\right)$. Moreover, $\Delta^{+}(D)=2 r-k$.

Theorem 2.1. For any digraph $D$ of order $n$,

$$
\gamma_{k}^{d}(D) \geq \frac{2 n}{\Delta^{+}-k+2}
$$

Moreover, the equality holds if and only if $D \in \Omega$.

Proof. Let $S$ be a $\gamma_{k}^{d}(D)$-set. Since $S$ is a dominating set and $\operatorname{deg}_{S}^{+}(v) \geq \operatorname{deg}_{\frac{S}{S}}^{+}(v)+k$ for all $v \in S$, we have

$$
\begin{aligned}
n-|S| \leq \sum_{v \in \bar{S}} \operatorname{deg}_{S}^{-}(v) & =\left|(S, \bar{S})_{D}\right|=\sum_{v \in S} \operatorname{deg}_{\bar{S}}^{+}(v) \leq \sum_{v \in S}\left(\operatorname{deg}_{S}^{+}(v)-k\right) \\
& =\sum_{v \in S}\left(\operatorname{deg}^{+}(v)-\operatorname{deg}_{\bar{S}}^{+}(v)-k\right) \\
& \leq-k|S|+\Delta^{+}|S|-n+|S| .
\end{aligned}
$$

Thus, $|S| \geq 2 n /\left(\Delta^{+}-k+2\right)$.

Let $D \in \Omega$. It is readily seen that $V\left(D^{\prime}\right)$ is a global defensive $k$-alliance in $D$. Since every vertex in $\overline{V\left(D^{\prime}\right)}$ is adjacent from exactly one vertex in $V\left(D^{\prime}\right)$, we have $n-\left|V\left(D^{\prime}\right)\right|=\left|\left(V\left(D^{\prime}\right), \overline{V\left(D^{\prime}\right)}\right)_{D}\right|$. Furthermore,

$$
\left|\left(V\left(D^{\prime}\right), \overline{V\left(D^{\prime}\right)}\right)_{D}\right|=\sum_{v \in V\left(D^{\prime}\right)} \operatorname{deg}_{\overline{V\left(D^{\prime}\right)}}^{+}(v)=\sum_{v \in V\left(D^{\prime}\right)}(r-k)=\left|V\left(D^{\prime}\right)\right|(r-k) .
$$

This shows that $\gamma_{k}^{d}(D) \leq\left|V\left(D^{\prime}\right)\right|=n /(r-k+1)=2 n /\left(\Delta^{+}-k+2\right)$, which implies the equality.

Suppose now that the equality holds and that $X$ is a $\gamma_{k}^{d}(D)$-set in the digraph $D$. Then, the inequalities in (2.1) hold with equality when we use $X$ instead of $S$. We let $D^{\prime}=D\langle X\rangle$. We first show that the out-degrees of the vertices of $D^{\prime}$ are the same. Suppose to the contrary that $\operatorname{deg}_{X}^{+}(u)<\Delta^{+}\left(D^{\prime}\right)$, for some $u$ in $X$. This yields to,

$$
\begin{aligned}
n-|X|=\left|(X, \bar{X})_{D}\right| & =-k|X|+\sum_{v \in X} \operatorname{deg}_{X}^{+}(v) \\
& =-k|X|+\sum_{v \in X} \operatorname{deg}^{+}(v)-(n-|X|) \\
& <-k|X|+\Delta^{+}|X|-n+|X| .
\end{aligned}
$$

So, $|X|>2 n /\left(\Delta^{+}-k+2\right)$, which is a contradiction. Therefore, all vertices of $D^{\prime}$ have the same out-degree $r$. Since $X$ is a defensive $k$-alliance in $D, \operatorname{deg}_{\frac{X}{X}}^{+}(v) \leq r-k$ for each $v \in X$. Taking into account this fact, and since $\sum_{v \in X} \operatorname{deg}_{\bar{X}}^{+}(v)=(r-k)|X|$, we have $\operatorname{deg}_{\bar{X}}^{+}(v)=r-k$ for each $v \in X$. On the other hand, since $X$ is a dominating set and $n-|X|=\left|(X, \bar{X})_{D}\right|$, every vertex in $\bar{X}$ has exactly one in-neighbor in $X$. Therefore, every vertex in $X$ has exactly $r-k$ private out-neighbors lying outside $X$. So, $D \in \Omega$. This completes the proof. by

We define the $k$-order-sum number of a digraph $D$ with non-increasing out-degree sequence $d_{1}^{+} \geq \ldots \geq d_{n}^{+}$

$$
o s n_{k}^{+}(D)=\min \left\{t \mid d_{1}^{+}+\ldots+d_{t}^{+} \geq 2 n+(k-2) t\right\} .
$$


Theorem 2.2. Let $D$ be a digraph of order $n$. Then, the following statements hold.

(i) $\gamma_{k}^{d}(D) \geq o s n_{k}^{+}(D) \geq \frac{2 n}{\Delta^{+}-k+2}$.

(ii) $\operatorname{osn}_{k}^{+}(D)=\frac{2 n}{\Delta^{+}-k+2}$ if and only if $2 n \equiv 0\left(\bmod \Delta^{+}-k+2\right)$ and $d_{s}^{+}=\Delta^{+}$, where $s=\frac{2 n}{\Delta^{+}-k+2}$.

Proof. (i) Let $S$ be a $\gamma_{k}^{d}(D)$-set. We have that $\operatorname{deg}_{S}^{+}(v) \geq \operatorname{deg}_{\bar{S}}^{+}(v)+k$, for all $v \in S$. Furthermore, $\sum_{v \in S} \operatorname{deg}_{\bar{S}}^{+}(v) \geq n-|S|$, since $S$ is a dominating set in $D$. This leads to

$$
\begin{aligned}
\sum_{v \in S} \operatorname{deg}^{+}(v) & =\sum_{v \in S} \operatorname{deg}_{S}^{+}(v)+\sum_{v \in S} \operatorname{deg}_{\bar{S}}^{+}(v) \\
& \geq \sum_{v \in S}\left(\operatorname{deg}_{\bar{S}}^{+}(v)+k\right)+\sum_{v \in S} \operatorname{deg}_{\frac{S}{S}}^{+}(v) \\
& \geq(n-|S|)+k|S|+(n-|S|) \\
& =2 n+(k-2)|S| .
\end{aligned}
$$

Therefore, $d_{1}^{+}+\ldots+d_{|S|}^{+} \geq 2 n+(k-2)|S|$ and so, $\gamma_{k}^{d}(D) \geq o s n_{k}^{+}(D)$. Now, let $t=o s n_{k}^{+}(D)$. Hence, the second inequality in (i) follows from

$$
t \Delta^{+} \geq d_{1}^{+}+\ldots+d_{t}^{+} \geq 2 n+(k-2) t .
$$

(ii) Let $\operatorname{osn}_{k}^{+}(D)=2 n /\left(\Delta^{+}-k+2\right)=s$. Note that $2 n \equiv 0\left(\bmod \Delta^{+}-k+2\right)$. If $d_{s}^{+}<\Delta^{+}$, then

$$
(s-1) \Delta^{+}+\Delta^{+}-1 \geq d_{1}^{+}+\ldots+d_{s}^{+} \geq 2 n+(k-2) s .
$$

Thus, $s \geq(2 n+1) /\left(\Delta^{+}-k+2\right)$, which is a contradiction. Therefore, $d_{s}^{+}=\Delta^{+}$.

Conversely, we have $d_{1}^{+}=\ldots=d_{s}^{+}=\Delta^{+}$. Hence, $d_{1}^{+}+\ldots+d_{s}^{+}=2 n \Delta^{+} /\left(\Delta^{+}-k+2\right)=2 n+2 n(k-$ $2) /\left(\Delta^{+}-k+2\right)=2 n+(k-2) s$. This implies that, osn $_{k}^{+}(D)=2 n /\left(\Delta^{+}-k+2\right)$.

The difference between $\operatorname{osn}_{k}^{+}(D)$ and $2 n /\left(\Delta^{+}-k+2\right)$ can be arbitrary large. In fact, we have the following stronger assertion: for any positive integer $b$ there exists a digraph $D$ of order $n$ for which $\operatorname{osn}_{k}^{+}(D)-\left\lceil 2 n /\left(\Delta^{+}-\right.\right.$ $k+2)\rceil=b$. To see this, we first suppose that $k \geq 0$. Let $D^{\prime}$ be a digraph of order $3 b-1$ for which $\operatorname{deg}^{+}(v)=k$, for all $v \in V\left(D^{\prime}\right)$. Let $D$ be obtained from $D^{\prime}$ by adding a new vertex $u$ with $k+1$ out-neighbors in $V\left(D^{\prime}\right)$. Clearly, $n=3 b$ and $\Delta^{+}=k+1$. Let $t=o s n_{k}^{+}(D)$. Then,

$$
d_{1}^{+}+\ldots+d_{t}^{+}=k+1+(t-1) k \geq 2 n+(k-2) t
$$

implies that $t \geq(2 n-1) / 2$ and hence $t=n=3 b$. Moreover, $2 n /\left(\Delta^{+}-k+2\right)=2 b$, and these two facts show the existence of $D$ claimed for this case.

We next suppose that $k \leq-1$. Let $n=(b+1)(2-k)-1$ and consider the directed star $S_{n}$. Clearly, $\left\lceil 2 n /\left(\Delta^{+}-k+1\right)\right\rceil=1$. Assuming that $t=\operatorname{osn}_{k}^{+}\left(S_{n}\right)$, we then have $n-1 \geq 2 n+(k-2) t$. Therefore, $t=\lceil(n+1) /(2-k)\rceil=b+1$, which leads to the claimed existence for this second case.

For the next result, we construct the family $\Theta$ of digraphs $D$ as follows. Consider the complete biorientation $\overleftrightarrow{K_{p}}$ of the complete graph $K_{p}$ with $p \geq k+1$. We add $p-k-1$ private out-neighbors lying outside $V\left(\overleftrightarrow{K_{p}}\right)$, for all $v \in V\left(\overleftrightarrow{K_{p}}\right)$. Let

$$
V(D)=V\left(\overleftrightarrow{K_{p}}\right) \cup\left(\bigcup_{v \in V\left(\overleftrightarrow{K_{p}}\right)} p n^{+}\left(v, V\left(\overleftrightarrow{K_{p}}\right)\right)\right)
$$

Note that some arcs might be among the vertices in $\overline{V\left(\overleftrightarrow{K_{p}}\right)}$ and some arcs $(u, v)$, for some $u \in \overline{V\left(\overleftrightarrow{K_{p}}\right)}$ and $v \in V\left(\overleftrightarrow{K_{p}}\right)$. 
Theorem 2.3. For any digraph $D$ of order $n$,

$$
\gamma_{k}^{d}(D) \geq \frac{k+\sqrt{k^{2}+4 n}}{2}
$$

The equality holds if and only if $D \in \Theta$.

Proof. Let $S$ be a $\gamma_{k}^{d}(D)$-set in $D$. Since $S$ is a dominating set and $\operatorname{deg}_{S}^{+}(v)-\operatorname{deg}_{\bar{S}}^{+}(v) \geq k$, we have

$$
\begin{aligned}
n-|S| & \leq \sum_{v \in \bar{S}} \operatorname{deg}_{S}^{-}(v)=\left|(S, \bar{S})_{D}\right|=\sum_{v \in S} \operatorname{deg}_{\bar{S}}^{+}(v) \\
& \leq \sum_{v \in S}\left(\operatorname{deg}_{S}^{+}(v)-k\right) \leq \sum_{v \in S}(|S|-k-1)=|S|(|S|-k-1) .
\end{aligned}
$$

Solving the inequality $(2.2)$ for $|S|$, we have $\gamma_{k}^{d}(D)=|S| \geq\left(\sqrt{4 n+k^{2}}+k\right) / 2$.

Let $D \in \Theta$ and $S=V\left(\overleftrightarrow{K_{p}}\right)$. Since $S$ is a dominating set and $\operatorname{deg}_{S}^{+}(v)=\operatorname{deg}_{\bar{S}}^{+}(v)+k$ for all $v \in S$, we deduce $S$ is a global defensive $k$-alliance in $D$. On the other hand, every vertex in $\bar{S}$ has exactly one in-neighbor in $S$ and every vertex in $S$ has exactly $|S|-k-1$ out-neighbors in $\bar{S}$. Therefore, the inequalities in (2.2) hold with equality. So, $\gamma_{k}^{d}(D) \leq|S|=\left(k+\sqrt{k^{2}+4 n}\right) / 2$, implying the desired equality.

Conversely, suppose that $\gamma_{k}^{d}(D)=\left(\sqrt{4 n+k^{2}}+k\right) / 2$ and let $S$ be a $\gamma_{k}^{d}(D)$-set. Thus, the inequalities in $(2.2)$ hold with equality, necessarily. So, every vertex in $\bar{S}$ has exactly one in-neighbor in $S$, every vertex in $S$ has exactly $|S|-k-1$ private out-neighbors in $\bar{S}$, and every vertex in $S$ is adjacent to the other vertices in it. Therefore $D \in \Theta$.

We next make use of the following well-known theorem of Turán from extremal graph theory in order to give another lower bound for $\gamma_{k}^{d}(D)$. To this end, we recall that a digraph is $(r+1)$-clique-free if its underlying graph has no an $(r+1)$-clique as a set of vertices.

Lemma 2.4 (Turán's Theorem, [17]). If $G$ is an $(r+1)$-clique-free graph of order $n$, then

$$
|E(G)| \leq \frac{r-1}{2 r} \cdot n^{2}
$$

with equality if and only if $G$ is the Turán's graph $T_{n, r}$ and $r$ divides $n$.

Consider the digraph $D^{\prime}$ as the complete biorientation of a complete $r$-partite graph $G$ with $p \geq k /(r-1)$ vertices in each partite set. We add $(r-1) p-k$ private out-neighbors lying outside $V\left(D^{\prime}\right)$ for any $v \in V\left(D^{\prime}\right)$. Let

$$
V(D)=V\left(D^{\prime}\right) \cup\left(\bigcup_{v \in V\left(D^{\prime}\right)} p n^{+}\left(v, V\left(D^{\prime}\right)\right)\right) .
$$

Add some new arcs among the vertices in $\overline{V\left(D^{\prime}\right)}$ and some $\operatorname{arcs}(u, v)$, for some $u \in \overline{V\left(D^{\prime}\right)}$ and $v \in V\left(D^{\prime}\right)$ such that the obtained digraph $D$ is $(r+1)$-clique-free. We define $\Phi$ as the family of all such digraphs $D$.

Theorem 2.5. Let $r \geq 2$ be an integer. If $D$ is an $(r+1)$-clique-free digraph of order $n$, then,

$$
\gamma_{k}^{d}(D) \geq \frac{(k-1) r}{2(r-1)}+\frac{1}{2(r-1)} \sqrt{(k-1)^{2} r^{2}+4 n r(r-1)} .
$$

Furthermore, the equality holds if and only if $D \in \Phi$. 
Proof. Let $S$ be a $\gamma_{k}^{d}(D)$-set. Since $S$ is a dominating set and $\operatorname{deg}_{S}^{+}(v) \geq \operatorname{deg}_{\frac{S}{S}}^{+}(v)+k$ for all $v \in S$, we have

$$
n-|S| \leq \sum_{v \in \bar{S}} \operatorname{deg}_{S}^{-}(v)=\left|(S, \bar{S})_{D}\right|=\sum_{v \in S} \operatorname{deg}_{\bar{S}}^{+}(v) \leq \sum_{v \in S} \operatorname{deg}_{S}^{+}(v)-k|S| .
$$

Taking into account the possible opposite arcs, by Lemma 2.4 we have

$$
\sum_{v \in S} \operatorname{deg}_{S}^{+}(v)=|A(D\langle S\rangle)| \leq \frac{r-1}{r}|S|^{2} .
$$

By considering together inequalities (2.3) and (2.4), we deduce $(r-1)|S|^{2} / r-(k-1)|S|-n \geq 0$. Solving this equation for $|S|$ we obtain the desired lower bound.

Assume first that the equality holds and that $S$ is a $\gamma_{k}^{d}(D)$-set. Hence, all inequalities in (2.3) and (2.4) hold with equality, necessarily. Now, Lemma 2.4 and (2.4) imply that $D\langle S\rangle=\overleftrightarrow{K_{p_{1}, \ldots, p_{r}}}$, in which case $p_{1}=$ $\ldots=p_{r}=|S| / r=p$. Moreover, the equalities in (2.3) show that every vertex of $D\langle S\rangle$ has $(r-1) p-k$ private out-neighbors lying outside of $V(D\langle S\rangle)$. Therefore, $D \in \Phi$.

Conversely, suppose that $D \in \Phi$. Clearly, $V\left(D^{\prime}\right)$ is a global defensive $k$-alliance in $D$ and $n=r p+r p((r-$ 1) $p-k)$. Therefore,

$$
\gamma_{k}^{d}(D) \leq\left|V\left(D^{\prime}\right)\right|=r p=\frac{(k-1) r}{2(r-1)}+\frac{1}{2(r-1)} \sqrt{(k-1)^{2} r^{2}+4 n r(r-1)},
$$

which implies the equality.

\section{RESUlts ON DiRECTED TREES WHEN $k=-1$}

In this section, we study the global defensive alliance number (i.e., the global defensive $k$-alliance number whether $k=-1$ ) of directed trees with emphasis on rooted trees. We first center our attention to rooted trees and let $r$ be the root of a rooted tree $T$. Suppose that $T_{1}=T$ and select a support vertex $u_{1}$ with maximum distance from $r$. Now let $T_{2}=T_{1}-N^{+}\left[u_{1}\right]$. Iterate this process, in which we always find a support vertex $u_{i}$ at a maximum distance from $r$ of the rooted tree $T_{i}=T_{i-1}-N^{+}\left[u_{i-1}\right]$. We end the process whether we have removed all vertices or get an isolated vertex. We consider the following situations.

- If the process described above ends with all the vertices removed, then we note that $\mathbb{P}_{1}=$ $\left\{N_{T_{1}}^{+}\left[u_{1}\right], \ldots, N_{T_{p}}^{+}\left[u_{p}\right]\right\}$ is a partition of $V(T)$, where $u_{1}, \ldots, u_{p}$ are the support vertices with maximum distance from $r$ in $T_{1}, \ldots, T_{p}$, respectively.

- If the process described above ends with the isolated vertex $r$ as the rooted tree $T_{p}$, then $\mathbb{P}_{2}=$ $\left\{N_{T_{1}}^{+}\left[u_{1}\right], \ldots, N_{T_{p-1}}^{+}\left[u_{p-1}\right],\{r\}\right\}$ is a partition of $V(T)$, where $u_{1}, \ldots, u_{p-1}$ are the support vertices with maximum distance from $r$ in $T_{1}, \ldots, T_{p-1}$, respectively.

In the process shown above we recursively eliminate a "special directed star" in each step, and obtain a sequence $T=T_{1}, \ldots, T_{p-1}, T_{p}$ of rooted trees. From now on we call it a recursive directed star elimination sequence (briefly RDSES). We define $\Pi$ as the family of all rooted trees $T$ such that each $\operatorname{RDSES} T=T_{1}, \ldots, T_{p-1}, T_{p}$ of it satisfies:

(a) at most one of $F_{p}=T_{p}$ and $F_{i-1}=T_{i-1}-T_{i}, 2 \leq i \leq p$, has even order;

(b) the central vertex $u_{i-1}$ of $F_{i-1}$, with even order $n_{i-1} \geq 4$, is adjacent to at most one central vertex among the central vertices of directed stars $F_{j}$;

(c) the central vertex $u_{i-1}$ of every $F_{i-1}$ with odd order has no out-neighbor among the central vertices of directed stars $F_{j}$. 
Theorem 3.1. Let $T$ be a rooted tree of order $n$. Then,

$$
\gamma_{-1}^{d}(T) \leq\left\lfloor\frac{n+\gamma(T)}{2}\right\rfloor
$$

The equality holds if and only if $T \in \Pi$.

Proof. Let $r$ be the root of $T$ and let $S$ be a $\gamma_{-1}^{d}(T)$-set. Consider an RDSES $T=T_{1}, \ldots, T_{p-1}, T_{p}$ of $T$. Assume that $u_{1}, \ldots, u_{p-1}$ and $u_{p}$ are the central vertices of $F_{1}, \ldots, F_{p-1}$ and $F_{p}=T_{p}$, respectively (note that if $r \in \bigcup_{i=1}^{p-1} V\left(T_{i}\right)$, then $F_{p}=T_{p}=\phi$ and thus the central vertex $u_{p}$ will not exist). Let $n_{i}=\left|V\left(F_{i}\right)\right|$, for $1 \leq i \leq p$.

Let $S^{\prime}$ be a $\gamma(T)$-set. Hence, there must be at least one vertex in $S^{\prime} \cap F_{i}$ for each $1 \leq i \leq p-1$. Moreover, $u_{p} \in S^{\prime}$ if $T_{p} \neq \phi$, necessarily. On the other hand, $\left\{u_{1}, \ldots, u_{p}\right\}\left(\left\{u_{1}, \ldots, u_{p-1}\right\}\right.$ when $\left.T_{p}=\phi\right)$ is a dominating set in $T$. This shows that the set of central vertices $u_{i}$ is a $\gamma(T)$-set. Without loss of generality, we assume that $S^{\prime}$ is such a set.

We construct $S^{\prime \prime}$ as the union of $S^{\prime}$ and a set containing $\left\lfloor\operatorname{deg}^{+}\left(u_{i}\right) / 2\right\rfloor$ vertices in $N^{+}\left(u_{i}\right)$, for all $1 \leq i \leq p$. Since $S^{\prime} \subseteq S^{\prime \prime}$, it follows $S^{\prime \prime}$ is a dominating set in $T$. Clearly, $\operatorname{deg}_{S^{\prime \prime}}(v) \geq \operatorname{deg}_{\overline{S^{\prime \prime}}}(v)-1$, for all $v \in S^{\prime}$. On the other hand, $N^{+}(v) \subseteq S^{\prime}$ for all $v \in S^{\prime \prime} \backslash S^{\prime}$. Thus, this inequality holds for all $v \in S^{\prime \prime}$. Therefore, $S^{\prime \prime}$ is a global defensive alliance in $T$. Let $I=\left\{i \mid 1 \leq i \leq p\right.$ and $n_{i}$ is odd $\}$. Hence,

$$
\gamma_{-1}^{d}(T) \leq\left|S^{\prime \prime}\right|=\sum_{i \notin I} \frac{n_{i}}{2}+\sum_{i \in I} \frac{n_{i}+1}{2}=\frac{n+|I|}{2} \leq \frac{n+\gamma(T)}{2},
$$

implying the desired upper bound.

Let $\gamma_{-1}^{d}(T)=\lfloor(n+\gamma(T)) / 2\rfloor$. Suppose, to a purpose of contradiction, that there exists an RDSES $T=$ $T_{1}, \ldots, T_{p-1}, T_{p}$ of $T$ in which at least two directed stars $F_{s}$ and $F_{t}$ have even orders. We now have $\gamma_{-1}^{d}(T) \leq$ $\frac{n+|I|}{2} \leq \frac{n+\gamma(T)-2}{2}$, by (3.1). Thus, $\gamma_{-1}^{d}(T)<\lfloor(n+\gamma(T)) / 2\rfloor$, which is a contradiction.

Now, suppose that for some RDSES $T=T_{1}, \ldots, T_{p-1}, T_{p}$ of $T$, the central vertex $u_{i}$ of a directed star $F_{i}$ with even order at least four is adjacent to two central vertices $u_{j}$ and $u_{j^{\prime}}$ of two directed stars $F_{j}$ and $F_{j^{\prime}}$, respectively. Thus, the set $S^{\prime \prime \prime}$ containing the members of $S^{\prime}$ along with $\left\lfloor\operatorname{deg}^{+}\left(u_{t}\right) / 2\right\rfloor$ out-neighbors for each $u_{t} \neq u_{i}$, and $\left\lfloor\operatorname{deg}^{+}\left(u_{i}\right) / 2\right\rfloor-1$ out-neighbors of $u_{i}$ is a global defensive alliance in $T$ and so, $\gamma_{-1}^{d}(T) \leq\left|S^{\prime \prime \prime}\right|<$ $\left|S^{\prime \prime}\right|=\lfloor(n+\gamma(T)) / 2\rfloor$, which is a contradiction again. Finally, a similar discussion shows that for any RDSES of $T$, the central vertices of the directed stars with odd orders have no out-neighbors among the central vertices.

Conversely, suppose that each RDSES of $T$ satisfies the conditions (a), (b) and (c). We now consider an RDSES $T=T_{1}, \ldots, T_{p-1}, T_{p}$ of $T$. If there exists a global defensive alliance $Q$ in $T$ for which $|Q|<\left|S^{\prime \prime}\right|$ and $S^{\prime \prime}$ as defined above from $T$, then there exists a directed star $F_{j}$ such that $1 \leq\left|Q \cap F_{j}\right|<\left|S^{\prime \prime} \cap F_{j}\right|=\left\lceil n_{j} / 2\right\rceil$. Therefore, $n_{j} \geq 3$ and $u_{j} \in Q$, necessarily. This implies that the central vertex $u_{j}$ is adjacent to a central vertex $u_{s}$ if $n_{j}$ is odd, and $u_{j}$ is adjacent to at least two central vertices $u_{s}$ and $u_{t}$ if $n_{j}$ is even. This contradicts our assumption that $T=T_{1}, \ldots, T_{p-1}, T_{p}$ satisfies (b) and (c). By this argument, we infer that

$$
\gamma_{-1}^{d}(T)=\left|S^{\prime \prime}\right|
$$

Let $n_{i}$ be odd, for all $1 \leq i \neq j \leq\left|S^{\prime}\right|$ (notice that $n_{j}$ can be either odd or positive even). So, $|I| \geq \gamma(T)-1$. Taking together (3.1) and (3.2) we deduce

$$
\gamma_{-1}^{d}(T)=\left|S^{\prime \prime}\right|=\frac{n+|I|}{2} \geq \frac{n+\gamma(T)-1}{2} .
$$

Therefore, $\gamma_{-1}^{d}(T) \geq\lfloor(n+\gamma(T)) / 2\rfloor$. This completes the proof.

We also define $\Sigma$ as the family of all directed trees $T$ obtained from a directed tree $T^{\prime}$ by adding $\operatorname{deg}_{T^{\prime}}(v)+1$ private out-neighbors lying outside $V\left(T^{\prime}\right)$, for each $v \in V\left(T^{\prime}\right)$. Furthermore, $V(T) \backslash V\left(T^{\prime}\right)$ is independent. 
Theorem 3.2. For any directed tree $T$ of order $n$,

$$
\gamma_{-1}^{d}(T) \geq \frac{n+1}{3}
$$

The equality holds if and only if $T \in \Sigma$.

Proof. Let $S$ be a $\gamma_{-1}^{d}(T)$-set and assume $T<S>$ has $c$ components. Then,

$$
-|S|+n-|S| \leq-|S|+\sum_{v \in S} \operatorname{deg}_{\bar{S}}^{+} \leq \sum_{v \in S} \operatorname{deg}_{S}^{+}(v)=|S|-c .
$$

Therefore,

$$
\gamma_{-1}^{d}(T)=|S| \geq \frac{n+c}{3} \geq \frac{n+1}{3} .
$$

Suppose now that $\gamma_{-1}^{d}(T)=(n+1) / 3$. We deduce from (3.5) that, $c=1$ and therefore $T^{\prime}=T<S>$ is a directed tree. By (3.4) we have $\operatorname{deg}_{S}^{+}(v)=-1+\operatorname{deg}_{\bar{S}}^{+}(v)$, for all $v \in S$. Moreover, $n-|S|=\sum_{v \in S} \operatorname{deg}_{\bar{S}}^{+}(v)$ by (3.4). Therefore, every vertex $v \in T^{\prime}$ has exactly $\operatorname{deg}_{T^{\prime}}^{+}(v)+1$ private out-neighbors lying outside $V\left(T^{\prime}\right)$. On the other hand, since $T$ is a directed tree and every vertex lying outside $V\left(T^{\prime}\right)$ has an in-neighbor in $V\left(T^{\prime}\right)$, $V(T) \backslash V\left(T^{\prime}\right)$ is independent. Thus, $T \in \Sigma$.

Conversely, assume that $T \in \Sigma$. Then, the inequalities in (3.4) and (3.5) hold with equality. Therefore, $\gamma_{-1}^{d}(T)=(n+1) / 3$.

Theorem 3.3. For any directed tree $T$ of order $n \geq 2, \gamma_{-1}^{d}(T) \geq o s n_{-1}^{+}(T) \geq(n+1) / 3$. Moreover, the equality holds if and only if $n \equiv 2(\bmod 3)$ and $d_{s}^{+}=0$ for $s>(n+1) / 3$.

Proof. Let $d_{1}^{+} \geq \ldots \geq d_{n}^{+}$be the non-increasing out-degree sequence of $T$ and $t=\operatorname{osn}_{-1}^{+}(T)$. Assume to the contrary that $t<(n+1) / 3$. Since $\sum_{v \in V(T)} \operatorname{deg}^{+}(v)=n-1$, we obtain that $n+1+n-1>3 t+d_{1}^{+}+\ldots+d_{t}^{+} \geq 2 n$, which is a contradiction. So, $o s n_{-1}^{+}(T) \geq(n+1) / 3$.

Let $\operatorname{ssn}_{-1}^{+}(T)=(n+1) / 3$. Obviously, $n \equiv 2(\bmod 3)$. On the other hand, $3(n+1) / 3+d_{1}^{+}+\ldots+d_{(n+1) / 3}^{+} \geq$ $2 n$ implies that, $\sum_{i=1}^{(n+1) / 3} d_{i}^{+}=n-1$. Thus, $d_{s}^{+}=0$ for $s>(n+1) / 3$. Conversely, by the hypotheses, $3(n+1) / 3+d_{1}^{+}+\ldots+d_{(n+1) / 3}^{+}=2 n$, which implies the equality.

In what follows we show that the difference between $o s n_{-1}^{+}(T)$ and $(n+1) / 3$ can be arbitrary large. That is, we claim that for any non-negative integer $b$ there exists a directed tree $T$ for which $\operatorname{osn}_{-1}^{+}(T)-(n+1) / 3=b$. To see this, consider $T=S_{6 b+2}^{-1}$. Hence, $3(3 b+1)+3 b+1=2(6 b+2)$. Therefore, $\operatorname{osn}_{-1}^{+}(T)=3 b+1$. So, $o s n_{-1}^{+}(T)-(n+1) / 3=b$.

\section{Computational issues}

We now continue our exposition by considering the problem of deciding whether the global defensive $k$-alliance number of a digraph is less than a given integer. That is stated in the following decision problem. In connection with this, one could immediately think into using the Remark 1.2, since there is such equivalence between defensive $k$-alliances in graphs and in digraphs. However, such remark becomes almost useless for our purpose, due to the fact that (surprisingly) it is not known the complexity of computing the global defensive $k$-alliance number of graphs, unless $k=-1$ (see $[7,19])$. 


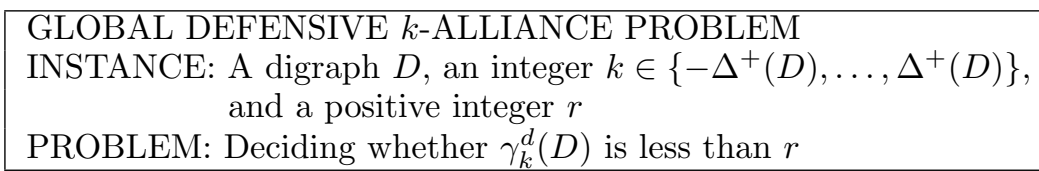

We next deal with the NP-completeness of the GLOBAL DEFENSIVE $k$-ALLIANCE PROBLEM (GD- $k$ A PROBLEM for short) above, for the non-negative interval of the value $k$ stated in the problem. In order to deal with this, we make a reduction from the 3-SAT problem, which is the most well known decision problem to be NP-complete (see [5]).

Theorem 4.1. For a digraph $D$ and an integer $k \in\left\{-1, \ldots, \Delta^{+}(D)\right\}$, the GD-kA PROBLEM is NP-complete.

Proof. The problem is easily seen to be in NP, since for a set of vertices $S$ of a digraph $D$, guessed by a nondeterministic algorithm for the problem, one can check in polynomial time that such set $S$ is indeed a global defensive $k$-alliance. The case $k=-1$ follows from the NP-completeness of the decision problem concerning computing the global defensive $k$-alliance number of graphs (see [7]) and the equivalence given in Remark 1.2. From now on, we assume $k \in\left\{0, \ldots, \Delta^{+}(D)\right\}$ and we describe a polynomial transformation of the 3-SAT problem to the GD- $k$ A PROBLEM.

Consider an arbitrary input $\mathcal{F}$ of the 3-SAT problem, given by a collection $C=\left\{c_{1}, c_{2}, \ldots, c_{m}\right\}$ of clauses over a finite set $U=\left\{u_{1}, u_{2}, \ldots, u_{n}\right\}$ of Boolean variables. We denote by $x_{i}$ and $\overline{x_{i}}$ the literals corresponding to the variable $u_{i}$, i.e., $x_{i}$ for a true assignment of $u_{i}$ and $\overline{x_{i}}$ for the false assignment. We shall construct a connected digraph $D_{F}=(V, A)$, such that setting a positive integer $r \leq|V|$, the digraph $D$ has a global defensive $k$-alliance of size at most $r$ if and only if $C$ is satisfiable.

For any variable $u_{i} \in U$ we create a digraph $D_{u_{i}}$ as follows. We begin with a complete biorientation of the complete graph $K_{k+2}$. Next, for any vertex $v \in V\left(K_{k+2}\right)$ we add a vertex $v^{\prime}$ and the $\operatorname{arc}\left(v, v^{\prime}\right)$. Then we add two extra vertices, denoted by $t_{u_{i}}$ and $f_{u_{i}}$ and add the $\operatorname{arcs}\left(x, t_{u_{i}}\right),\left(x, f_{u_{i}}\right),\left(t_{u_{i}}, x\right)$ and $\left(f_{u_{i}}, x\right)$ for every $x \in V\left(K_{k+2}\right)$. On the other hand, for every clause $c_{j} \in C$ we create a digraph $D_{c_{j}}$ as follows. We again begin with a complete biorientation of the complete graph $K_{k+1}$ and, also, for any vertex $v \in V\left(K_{k+1}\right)$ we add a vertex $v^{\prime}$ and the $\operatorname{arc}\left(v, v^{\prime}\right)$. In addition, we add a vertex $q_{c_{j}}$ and the $\operatorname{arcs}\left(q_{c_{j}}, x\right)$ and $\left(x, q_{c_{j}}\right)$ for every $x \in V\left(K_{k+1}\right)$. Finally, if $x_{i} \in c_{j}$ or $\overline{x_{i}} \in c_{j}$ for some $i \in\{1, \ldots, n\}$ and $j \in\{1, \ldots, m\}$, then we add the arc $\left(q_{c_{j}}, t_{u_{i}}\right)$ or $\left(q_{c_{j}}, f_{u_{i}}\right)$, respectively. A fairly representative example of a subdigraph of a digraph $D_{F}$ is given in Figure 1 by taking $k=2$.

We will show that $\mathcal{F}$ is satisfiable if and only if $\gamma_{k}^{d}\left(D_{F}\right)=n(k+3)+m(k+2)$. We first observe the following claim.

Claim 1: The digraph $D_{F}$ satisfies that $\gamma_{k}^{d}\left(D_{F}\right) \geq n(k+3)+m(k+2)$.

Proof of Claim 1: In order to carry out the domination condition, we readily note that every vertex belonging to a subdigraph $K_{k+2}$ used to generate a gadget corresponding to a variable and every vertex belonging to a subdigraph $K_{k+1}$ used to generate a gadget corresponding to a clause belong to every global defensive $k$-alliance in $D_{F}$. Moreover, in order to achieve the global defensive $k$-alliance condition, at least one vertex of the pair $t_{u_{i}}, f_{u_{i}}$, for every $i \in\{1, \ldots, n\}$, and the vertex $q_{c_{j}}$, for every $j \in\{1, \ldots, m\}$, must be included in a given global defensive $k$-alliance. Thus, the lower bound follows. $(\square)$

We next continue our reduction by separating it into a necessary and a sufficient situation.

$(\Rightarrow)$ Assume we have a satisfiable assignment for $\mathcal{F}$. Let $S$ be a set of vertices in $D_{F}$ given as follows.

- Any vertex belonging to a subdigraph $K_{k+2}$ used to generate a gadget corresponding to a variable is included in $S$.

- Any vertex belonging to a subdigraph $K_{k+1}$ used to generate a gadget corresponding to a clause is included in $S$.

- Any vertex $q_{c_{j}}$ with $j \in\{1, \ldots, m\}$ is included in $S$.

- If $u_{i} \in U$ has assigned the value TRUE, then we add to $S$ the vertex $t_{u_{i}}$. 


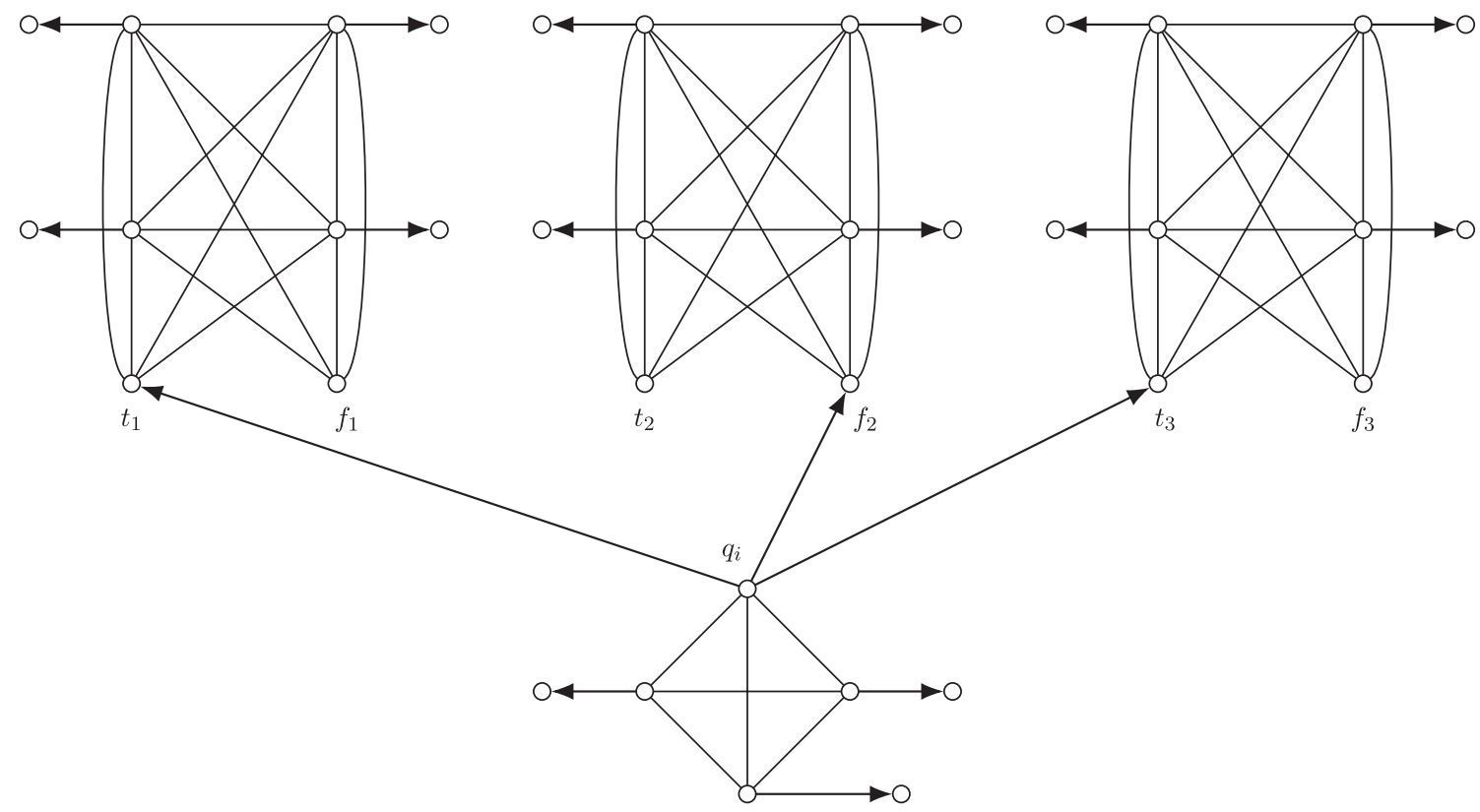

FIgURE 1 . The subdigraph corresponding to a clause $c_{i}=\left(u_{1}, \overline{u_{2}}, u_{3}\right)$ by taking $k=2$. An edge without arrows means there exists an arc in both directions.

- If $u_{i} \in U$ has assigned the value FALSE, then we add to $S$ the vertex $f_{u_{i}}$.

Note that the cardinality of $S$ is precisely $n(k+3)+m(k+2)$. We can readily see that $S$ is a dominating set. Moreover, the global defensive $k$-alliance condition is satisfied for every vertex of $S$. That is,

- if $x \in V\left(K_{k+2}\right)$ for some subdigraph used to generate a gadget corresponding to a variable, then $\operatorname{deg}_{S}^{+}(x)=k+2=\operatorname{deg}_{\bar{S}}^{+}(x)+k$;

- if $x \in V\left(K_{k+1}\right)$ for some subdigraph used to generate a gadget corresponding to a clause, then $\operatorname{deg}_{S}^{+}(x)=$ $k+1=\operatorname{deg}_{\bar{S}}^{+}(x)+k$;

- if $x=t_{u_{i}}$ or $x=f_{u_{i}}$ for some $i \in\{1, \ldots, n\}$, then $\operatorname{deg}_{S}^{+}(x)=k+2>k=\operatorname{deg}_{\bar{S}}^{+}(x)+k$;

- if $x=q_{c_{j}}$ for some $j \in\{1, \ldots, m\}$, then since $\mathcal{F}$ is satisfiable, the clause $c_{j}$ must contain at least one literal $x_{l}$ or $\overline{x_{l}}$ which is satisfied. This means that

- $u_{l}$ has assigned the value TRUE (whether $c_{j}$ contains the literal $x_{l}$ ) and the vertex $t_{u_{l}} \in S$, or

- $u_{l}$ has assigned the value FALSE (whether $c_{j}$ contains the literal $\overline{x_{l}}$ ) and the vertex $f_{u_{l}} \in S$.

In any case we obtain that $\operatorname{deg}_{S}^{+}(x) \geq k+2$ and $\operatorname{deg}_{S}^{+}(x) \leq 2$, which leads to $\operatorname{deg}_{S}^{+}(x) \geq k+2 \geq \operatorname{deg}_{\bar{S}}^{+}(x)+k$.

Thus, $S$ is a global defensive $k$-alliance in $D_{F}$ and so, $\gamma_{k}^{d}\left(D_{F}\right) \leq n(k+3)+m(k+2)$. Consequently, by Claim 1, we obtain the desired equality.

$(\Leftarrow)$ Assume $\gamma_{k}^{d}\left(D_{F}\right)=n(k+3)+m(k+2)$ and let $A$ be a $\gamma_{k}^{d}\left(D_{F}\right)$-set. By using the ideas of the short proof of Claim 1, we see that $A$ contains every vertex belonging to a subdigraph $K_{k+2}$ used to generate a gadget corresponding to a variable and every vertex belonging to a subdigraph $K_{k+1}$ used to generate a gadget corresponding to a clause. Also, $A$ contains at least one vertex of the pair $t_{u_{i}}, f_{u_{i}}$, for every $i \in\{1, \ldots, n\}$, and the vertex $q_{c_{j}}$, for every $j \in\{1, \ldots, m\}$. Since $\gamma_{k}^{d}\left(D_{F}\right)=n(k+3)+m(k+2)$, it must clearly happen that $A$ contains exactly one vertex of each pair $t_{u_{i}}, f_{u_{i}}$, for every $i \in\{1, \ldots, n\}$. Thus, we make the following assignment to the variables of $U$. If $t_{u_{i}} \in A$, then we assign to $u_{i}$ the value TRUE. Otherwise, if $f_{u_{i}} \in A$, then we assign to $u_{i}$ the value FALSE. We will show now that this is satisfiable assignment for the formula $\mathcal{F}$. Let $c_{j} \in C$ be any clause of $\mathcal{F}$. Note that, since the vertex $q_{c_{j}} \in A$ must satisfy the 
global defensive $k$-alliance condition, there exists a vertex $y \in\left\{t_{u_{i}}, f_{u_{i}}\right\}$ for some $u_{i} \in U$ such that $y$ is adjacent from $q_{c_{j}}$ and $y \in A$ (notice that exactly one vertex of the pair $t_{u_{i}}, f_{u_{i}}$ is in $A$, as stated above). If $y=t_{u_{i}}$, then $x_{i} \in c_{j}$ and $u_{i}$ has assigned the value TRUE, which means $c_{j}$ is satisfied. On the contrary, if $y=f_{u_{i}}$, then $\overline{x_{i}} \in c_{j}$ and $u_{i}$ has assigned the value FALSE, which means that again $c_{j}$ is satisfied. Since $c_{j}$ is arbitrarily taken, we deduce that every clause is satisfied, and therefore, $\mathcal{F}$ is satisfiable by our assignment, which complete the NP-completeness reduction.

As a consequence of the reduction above concerning the GD- $k$ A PROBLEM, we obtain the following result.

Corollary 4.2. For a digraph $D$ and an integer $k \in\left\{-1, \ldots, \Delta^{+}(D)\right\}$, the problem of computing the global defensive $k$-alliance number of $D$ is NP-hard.

Based on these two results above and with the knowledge of analogous results on alliances in graphs, one could naturally pose the following conjecture.

Conjecture 4.3. For a digraph $D$, the GD- $k$ A PROBLEM is NP-complete, and so, computing the global defensive $k$-alliance number of $D$ is NP-hard for any $k \in\left\{-\Delta^{+}(D), \ldots, \Delta^{+}(D)\right\}$.

Despite the GD- $k$ A PROBLEM would be proved to be NP-complete for any integer value $k \in$ $\left\{-\Delta^{+}(D), \ldots, \Delta^{+}(D)\right\}$, there are always several situations in which the problem becomes "easier", namely polynomial. One example, whether $k=-1$ and $D$ is a rooted tree, is next studied.

By a process similar to that one presented at the beginning of Section 3, we are able to provide an algorithm for finding a $\gamma_{-1}^{d}(T)$-set, for any rooted tree $T$, as follows. The algorithm, which is next stated, uses the so-called Breadth-First Search (BFS for short) algorithm $([10,20])$ for traversing the vertices of the underling tree of the rooted tree $T$. In the algorithm, given a vertex $v$, by $p(v)$ we mean the parent of $v$, and by $C h(v)$, the children of $v$ (see [18]).

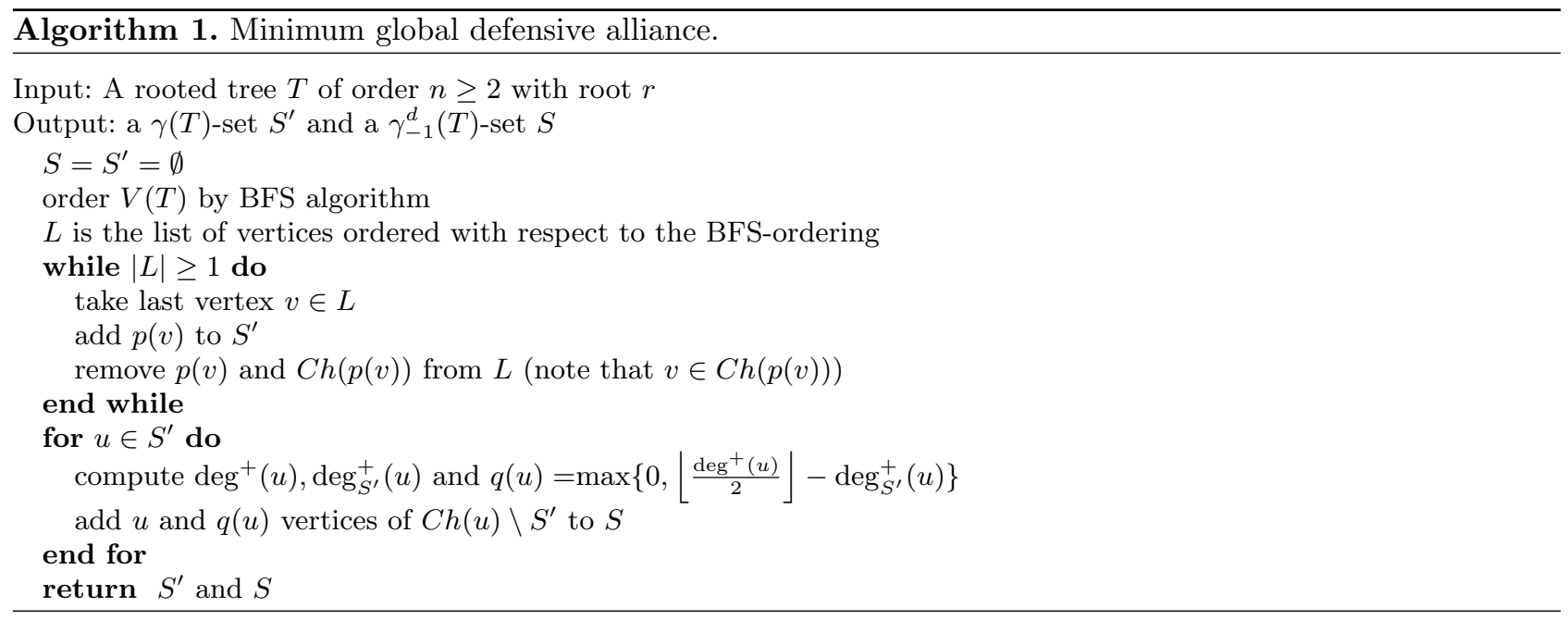

The following theorem ensures that the outputs of Algorithm 1. will always attain the minimum values for a global defensive alliance in a rooted tree $T$.

Theorem 4.4. For any rooted tree $T$ the subsets $S^{\prime}$ and $S$ constructed by Algorithm 1. are $\gamma(T)$ and $\gamma_{-1}^{d}(T)$ sets, respectively. 
Proof. As we showed in the proof of Theorem 3.1 the subset $S^{\prime}$ is a $\gamma(T)$-set. Clearly, $S$ is a dominating set in $T$. For any $u \in S^{\prime}, \operatorname{deg}_{S}^{+}(u)=q(u)+\operatorname{deg}_{S^{\prime}}^{+}(u) \geq\left\lfloor\operatorname{deg}^{+}(u) / 2\right\rfloor$. On the other hand, $N^{+}(v) \subseteq S^{\prime} \subseteq S$ for each $v \in S \backslash S^{\prime}$. Therefore, $S$ is a global defensive alliance in $T$. Now let $A$ be a $\gamma_{-1}^{d}(T)$-set. Then $A$ intersects $\{u\} \cup C h(u)$ at greater or equal than $1+q(u)$ vertices, for each $u \in S^{\prime}$. So, $|S| \leq|A|$. Thus, $|S|=\gamma_{-1}^{d}(T)$.

Since it is well known that the BFS algorithm runs in linear time for trees, and according to the previously described process, it is easy to check that Algorithm 1. can be implemented to run in polynomial-time on the order of the rooted tree $T$.

\section{CONCluding REMARKS AND FUTURE WORK}

- As in the case of graphs, alliances can be analyzed not in a global way, but in a local one. In this sense, one can define the following parameter for a digraph $D$. A set of vertices $S \subseteq V(D)$ is called a defensive $k$-alliance in $D$ provided that $\operatorname{deg}_{S}^{+}(v) \geq \operatorname{deg}_{\bar{S}}^{+}(v)+k$ for all $v \in S$ (notice that we do not consider now the domination property). The defensive $k$-alliance number, which could be denoted $a_{k}^{d}(D)$, is defined as the minimum cardinality of a defensive $k$-alliance in $D$. In consequence, the study of the not global case for a defensive $k$-alliance is clearly of potential interest to continue this research line.

- Similarly to the case of graphs, where offensive alliances (global and not global) are also defined and studied, for digraph an analogous parameter can be presented. That is, a set of vertices $S \subseteq V(D)$ is called a global offensive $k$-alliance in $D$ provided that every vertex in $V(D) \backslash S$ is adjacent from a vertex in $S$ and $\operatorname{deg}_{S}^{-}(v) \geq \operatorname{deg}_{\bar{S}}^{-}(v)+k$, for all $v \in V(D) \backslash S$. The global offensive $k$-alliance number, denoted $\gamma_{k}^{o}(D)$, is defined as the minimum cardinality of a global offensive $k$-alliance in $D$. Results on global offensive $k$-alliances in digraphs shall be given in the ongoing work [9]. Notice that, the definition of offensive alliances is more natural while the condition regards in-degrees, in order that the "offensive" property of the set in question will make sense. Clearly, the local version of offensive alliances is also of interest to continue developing this research line.

- An interesting question concerning this work concerns completing the NP-hardness property of computing the global defensive $k$-alliance number of digraphs. That is, proving the Conjecture 4.3. Moreover, it would be interesting to find some classes of digraphs whether such problem could be polynomially solved. For instance, we have shown that finding global defensive $k$-alliances in rooted trees can be solved in polynomial time. Can such result be generalized to directed trees in general?

- A final remark is again related with studying an alliance variant equivalent to the one from not directed graphs. That is the case of (global) powerful $k$-alliances. In a graph $G$, a set of vertices $S \subset V(G)$ is a (global) powerful $k$-alliance if it is a (global) defensive $k$-alliance and a (global) offensive $(k+2)$-alliance. It is then natural to consider the (global) powerful $k$-alliances in digraphs, which can be defined in a similar way, and study their mathematical properties.

Acknowledgements. We are grateful to the anonymous referees for their helpful comments and suggestions that resulted in a better organized paper.

\section{REFERENCES}

[1] J. Bang-Jensen and G. Gutin, Digraphs: Theory, Algorithms and Applications. Springer Monographs in Mathematics. SpringerVerlag London Ltd., London (2007).

[2] H. Fernau and J.A. Rodríguez-Velázquez, A survey on alliances and related parameters in graphs. Electron. J. Graph Theory Appl. 2 (2014) 70-86.

[3] G.W. Flake, S. Lawrence and C.L. Giles, Efficient identification of web communities. In: Proceedings of the sixth ACM SIGKDD international conference on Knowledge discovery and data mining. KDD '00, ACM, New York, NY, USA (2000).

[4] Y. Fu, Dominating set and converse dominating set of a directed graph. Am. Math. Mon. 75 (1968) 861-863.

[5] M.R. Garey and D.S. Johnson, Computers and Intractability: A Guide to the Theory of NP-Completeness. W. H. Freeman \& Co., New York, USA (1979).

[6] T.W. Haynes, S.T. Hedetniemi and P.J. Slater, Fundamentals of Domination in Graphs. Marcel Dekker, New York, NY (1998). 
[7] L.H. Jamieson, Algorithms and complexity for alliances and weighted alliances of varoius types. Ph.D. thesis, Clemson University, Clemson, SC, USA (2007).

[8] P. Kristiansen, S.M. Hedetniemi and S.T. Hedetniemi, Alliances in graphs. J. Combin. Math. Combin. Comput. 48 (2004) $157-177$.

[9] D.A. Mojdeh, B. Samadi and I.G. Yero, Global offensive $k$-alliances in digraphs. Preprint arXiv: 1905.01259 [math.CO] (2019).

[10] E.F. Moore, The shortest path through a maze. In: Proceedings of the International Symposium on the Theory of Switching. Harvard University Press (1959) 285-292.

[11] K. Ouazine, H. Slimani and A. Tari, Alliances in graphs: parameters, properties and applications-A survey. AKCE Int. J. Graphs Comb. 15 (2018) 115-154.

[12] J.A. Rodríguez-Velázquez and J.M. Sigarreta, Global defensive k-alliances in graphs. Discrete Appl. Math. 157 (2009) $211-218$.

[13] K.H. Shafique, Partitioning a graph in alliances and its application to data clustering. Ph.D. thesis, University of Central Florida (2004).

[14] K.H. Shafique and R.D. Dutton, Maximum alliance-free and minimum alliance-cover sets. Congr. Numer. 162 (2003) $139-146$.

[15] K.H. Shafique and R.D. Dutton, A tight bound on the cardinalities of maximun alliance-free and minimun alliance-cover sets. J. Combin. Math. Combin. Comput. 56 (2006) 139-145.

[16] K.H. Shafique and R.D. Dutton, On satisfactory partitioning of graphs. Congr. Numer. 154 (2002) $183-194$.

[17] P. Turán, On an extremal problem in graph theory. Math. Fiz. Lapok. 48 (1941) 436-452.

[18] D.B. West, Introduction to Graph Theory, 2nd edition. Prentice Hall, USA (2001).

[19] I.G. Yero and J.A. Rodríguez-Velázquez, A survey on alliances in graphs: defensive alliances. Utilitas Math. 105 (2017) $141-172$.

[20] K. Zuse, Der Plankalkül (in German), Konrad Zuse Internet Archive (1972) 96-105. 